\title{
Dengue dynamics and vaccine cost-effectiveness in Brazil
}

\author{
David P. Durham, $\mathrm{PhD}^{\star}, 1$, Martial L. Ndeffo Mbah, $\mathrm{PhD}^{1}$, Jan Medlock, $\mathrm{PhD}^{2}$, Paula M. Luz, \\ $\mathbf{M D}^{3}$, Lauren A. Meyers, $\mathbf{P h D}^{4}$, A. David Paltiel, $\mathbf{P h D}^{1}$, and Alison P. Galvani, $\mathbf{P h D}^{1}$ \\ ${ }^{1}$ School of Public Health, Yale University, New Haven, CT, United States \\ ${ }^{2}$ Department of Biomedical Sciences, Oregon State University, Corvallis, OR, United States \\ ${ }^{3}$ Evandro Chagas Clinical Research Institute, Oswaldo Cruz Foundation, Rio de Janeiro, Brazil \\ ${ }^{4}$ Section of Integrative Biology, University of Texas at Austin, Austin, TX, United States
}

\begin{abstract}
Recent Phase $2 \mathrm{~b}$ dengue vaccine trials have demonstrated the safety of the vaccine and estimated the vaccine efficacy with further trials underway. In anticipation of vaccine roll-out, costeffectiveness analysis of potential vaccination policies that quantify the dynamics of disease transmission are fundamental to the optimal allocation of available doses.

We developed a dengue transmission and vaccination model and calculated, for a range of vaccination costs and willingness-to-pay thresholds, the level of vaccination coverage necessary to sustain herd-immunity, the price at which vaccination is cost-effective and is cost-saving, and the sensitivity of our results to parameter uncertainty. We compared two vaccine efficacy scenarios, one a more optimistic scenario and another based on the recent lower-than-expected efficacy from the latest clinical trials.
\end{abstract}

We found that herd-immunity may be achieved by vaccinating $82 \%$ (95\% CI 58-100\%) of the population at a vaccine efficacy of $70 \%$. At this efficacy, vaccination may be cost-effective for vaccination costs up to US \$534 (95\% CI \$369-1008) per vaccinated individual and cost-saving up to $\$ 204$ (95\% CI \$39-678). At the latest clinical trial estimates of an average of 30\% vaccine efficacy, vaccination may be cost-effective and cost-saving at costs of up to $\$ 237$ (95\% CI $\$ 159$ 512) and \$93 (95\% CI \$15-368), respectively.

Our model provides an assessment of the cost-effectiveness of dengue vaccination in Brazil and incorporates the effect of herd immunity into dengue vaccination cost-effectiveness. Our results demonstrate that at the relatively low vaccine efficacy from the recent Phase $2 b$ dengue vaccine trials, age-targeted vaccination may still be cost-effective provided the total vaccination cost is sufficiently low.

\section{Keywords}

Dengue; vaccine; modeling; cost-effectiveness

\footnotetext{
(c) 2013 Elsevier Ltd. All rights reserved.

* Corresponding author. School of Public Health, Yale University, New Haven, CT, United States. david.durham@yale.edu, 412-417-5732.
}

Publisher's Disclaimer: This is a PDF file of an unedited manuscript that has been accepted for publication. As a service to our customers we are providing this early version of the manuscript. The manuscript will undergo copyediting, typesetting, and review of the resulting proof before it is published in its final citable form. Please note that during the production process errorsmaybe discovered which could affect the content, and all legal disclaimers that apply to the journal pertain. 


\section{Introduction}

Dengue poses a significant threat to both public health and economic progress in many regions of the world $(1,2)$. In Brazil, there are more than two million dengue fever cases annually resulting in an economic burden of over US $\$ 1$ billion (2). Three serotypes of the virus (DENV-1, DENV-2, DENV-3) are currently endemic in Brazil, with the fourth serotype (DENV-4) emerging $(3,4)$.

Vaccine development has been challenging due to the necessity of eliciting long-lived protection against all four serotypes in order to avoid the elevated disease severity associated with secondary infections $(5,6)$. While initial studies indicated that a three dose-regime of the Sanofi Pasteur vaccine would elicit protective immunity against all four serotypes $(7,8)$, the latest vaccine trial points to a lower overall efficacy with no evidence of serotypespecific protection against DENV-2 (9). However, sample-size biases may have arisen in these trials (10), and further vaccine efficacy studies are underway. Recently several promising candidate tetravalent vaccines have been developed, with availability projected by 2015-2020 (11,12).

As vaccine development nears completion, country-specific studies that quantify the costeffectiveness of the vaccine will be critical to successful public health strategies. Previous studies have estimated the cost-effectiveness of a hypothetical dengue vaccine in Southeast Asia (13-16). However, dengue vaccine cost-effectiveness has yet to be evaluated in Brazil, where costs and epidemiological dynamics are distinct from those of Southeast Asia. Furthermore, dengue vaccine cost-effectiveness models have not addressed the secondary effects of vaccination on reducing transmission beyond the vaccine recipients (17). We have previously shown that the effectiveness associated with vaccination campaigns can be substantially under-estimated if secondary transmission effects are not considered (18).

Here, we evaluated the cost-effectiveness of dengue vaccination, taking into account transmission dynamics, and compared the range of clinical estimates from the latest trials with more optimistic efficacies suggested by initial trials of the Sanofi Pasteur vaccine and by other promising vaccine candidates (6-8). We calculated the level of routine childhood coverage required to maintain herd-immunity and evaluated a range of vaccination policies that are currently under discussion $(12,14,19)$. We evaluated the costs associated with, and disability-adjusted life years (DALYs) saved by, alternative vaccination policies (20), and estimated the vaccination cost at which vaccination satisfies internationally accepted standards as cost-effective, very cost-effective, and cost-saving. We evaluated the sensitivity of our results to parameter uncertainty across a range of vaccination costs and willingnessto-pay thresholds.

\section{Methods}

We developed a deterministic, age-structured four-serotype dengue transmission model (described in the Supplement). We evaluated 400 potential vaccination policies, each consisting of 1) between 0 and $90 \%$ routine childhood coverage, in increments of $10 \%, 2$ ) a one-time mass vaccination, as the vaccine becomes available, of one of four overlapping, increasingly inclusive age groups: $0-5$ year olds, $0-15$ year olds, $0-40$ year olds, or the full population, and 3) a level of one-time mass vaccination between 0 and $90 \%$, in steps of $10 \%$. We modeled the routine childhood coverage by transitioning a fraction $\Phi v_{i}$ of young children into the vaccinated class following maternal antibody waning, where $\Phi$ represents the vaccine efficacy. We modeled the one-time mass vaccination by transitioning a fraction $\Phi v_{a}$ of all individuals in age group $a$ into the vaccinated class at the start of the simulation. 
We compared a relatively optimistic vaccine efficacy of 70\% (49-87\%) with a lower vaccine efficacy based on the Phase $2 b$ trial results of $30 \%$ (95\% CI -13.4-56.6\%) (9).

\section{Population herd-immunity}

To determine the degree of vaccine coverage necessary to halt transmission and sustain herd-immunity, which we numerically estimated as occurring when dengue incidence was under $1 \%$ of the pre-vaccination incidence, we assumed a constant fraction $v_{i}$ of young children are vaccinated and calculated the incidence at the endemic equilibrium while varying $v_{i}$ from 0 to 1 . To calculate the $95 \%$ credible interval of herd-immunity, we defined parameter distributions for the input parameters (Table S1), and conducted 10,000 Monte Carlo iterations for each level $v_{i}$.

\section{Efficient and cost-effective vaccination policies}

Efficient policies are defined as those which produce the greatest DALYs saved for a given outlay. To identify efficient policies, we calculated the time-discounted DALYs lost to dengue fever, dengue hemorrhagic fever / dengue shock syndrome, and dengue-related deaths, and the total costs accrued due to vaccination, medical treatment, and lost productivity over 73 years, the life expectancy in Brazil (21). To determine the net DALYs saved, we subtracted the total DALYs lost across the population under the vaccination scenario from the total DALYs lost across the population under the no vaccination scenario. To determine the net costs accrued, we subtracted the total costs accrued under the no vaccination scenario from the total costs accrued under the vaccination scenario. We varied the vaccination cost inclusive of the full three-dose regimen and the costs of vaccine delivery and administration, drawing from estimates of the anticipated costs of dengue vaccine production, over the range $\$ 10-300(13,15,16,19)$.

\section{Cost-effective, very cost-effective, and cost-saving vaccination costs}

To identify the threshold costs at which vaccination becomes cost-effective and very costeffective, we calculated the net benefit of every policy, defined as (DALYs saved) minus (net cost divided by willingness-to-pay) (22). Interventions that had a positive net benefit at a willingness-to-pay of three times the per capita $\operatorname{GDP}(\$ 36,000)$ were deemed "costeffective", and those that had positive net benefits at one times the per capita GDP $(\$ 12,000)$ were deemed "very cost-effective", in accordance with the WHO Commission on Macroeconomics and Health $(21,23-25)$. To derive the cost-effective and the very costeffective vaccination costs, we identified the maximum vaccination cost at which there is at least one policy with positive net benefits at the two willingness-to-pay thresholds. To derive the threshold at which vaccination becomes cost-saving, we calculated the maximum vaccination cost at which there is at least one policy with a negative net discounted cost.

To calculate the $95 \%$ credible intervals for the cost-effective, very cost-effective, and costsaving vaccination costs, we generated 10,000 samples from a distribution of the societal costs of dengue fever and dengue hemorrhagic fever / dengue shock syndrome in Brazil (Table S2) (1).

\section{Sensitivity to parameter uncertainty}

To evaluate the sensitivity of our results to parameter uncertainty, we compared five vaccination policies that are consistent with estimated rates of childhood vaccination schedule completion in Rio de Janeiro and contain increasingly aggressive levels of one time mass vaccination (26): 1) $80 \%$ routine childhood coverage, 2) $80 \%$ routine childhood coverage and mass vaccination of $50 \%$ of $0-5$ year olds, 3) $80 \%$ routine childhood coverage and mass vaccination of $50 \%$ of $0-15$ year olds, 4) $80 \%$ routine childhood coverage and 
mass vaccination of 50\% of 0-40 year olds, and 5) $80 \%$ routine childhood coverage and mass vaccination of $50 \%$ of the entire population. We defined probability distributions for each parameter (Tables S1 and S2) and randomly sampled values from these distributions to generate 10,000 independent model outcomes. We calculated the probability for each policy of having the greatest net benefit at the cost-effective threshold and at the very cost-effective threshold as vaccination cost was increased.

To evaluate the sensitivity of our findings to vaccination cost, we assigned to vaccination cost a uniform uncertainty distribution of \$10-300 and calculated the probability for each policy of having the greatest net benefit as willingness-to-pay was increased from $\$ 0$ to $\$ 36,000$ (the cost-effective threshold).

\section{Phase $2 \mathrm{~b}$ dengue vaccine efficacy}

To evaluate the vaccination policies under the lower rates of vaccine efficacy estimated during recent Phase $2 \mathrm{~b}$ dengue vaccine trials, we repeated all simulations using a vaccine efficacy defined by a Normal distribution, with a mean of $30 \%$ and a standard deviation of $13 \%$, constrained to the range $[0 \%, 100 \%]$, approximating the reported vaccine efficacy of $30 \%(95 \% \mathrm{CI}-13.4-56.6 \%)(9)$.

\section{Results}

\section{Population herd-immunity}

We calculated an equilibrium annual dengue infection incidence in the absence of vaccination of $4.56 \%$ and an annual symptomatic dengue fever incidence of $1.06 \%$, comparable to empirical estimates $(2,27,28)$. We found that herd-immunity may be achieved, at $70 \%$ vaccine efficacy, by routine childhood coverage of at least $82 \%$, although the upper bound of the $95 \%$ credible interval exceeded $100 \%$ (Figure 1).

\section{Efficient and cost-effective vaccination policies}

We considered the net costs and DALYs saved across 400 unique vaccination policies. At an example cost of $\$ 250$, most of those policies that are efficient, saving the most DALYs for a given outlay, and cost-effective, saving at least as many DALYs as the net cost divided by the willingness-to-pay, include between $60 \%$ and $80 \%$ routine childhood coverage, slightly below the herd-immunity level of $82 \%$ (Figure $2 \mathrm{c}$ ). While policies that include routine childhood coverage substantially below this level are efficient in some cases, policymakers may prefer larger programs that still fall below the willingness-to-pay but save many more DALYs (Figure 2a-b). Conversely, policies that include routine childhood coverage in excess of herd-immunity may be efficient, but are not cost-effective at the Brazilian willingness-to-pay threshold (Figure 2d). These results remain qualitatively similar across the full range of vaccination costs $(\$ 10-300)$, with the majority of efficient and costeffective policies across this range including between 60 and $80 \%$ routine childhood coverage.

\section{Cost-effective, very cost-effective, and cost-saving vaccination costs}

At a 70\% vaccine efficacy, we found that the cost-effective threshold occurs at \$534 (95\% CI \$369-1008), the very cost-effective threshold at $\$ 314$ (95\% CI \$149-788), and the costsaving threshold at \$204 (95\% CI \$39-678).

\section{Sensitivity to parameter uncertainty}

We compared five vaccination policies combining $80 \%$ routine childhood coverage with varying levels of one-time mass vaccination. We calculated the vaccination cost range over 
which each policy would be preferred, having the highest probability of having the greatest net benefits. We found $80 \%$ routine childhood coverage with mass vaccination of $50 \%$ of the entire population to be preferred at vaccination costs up to $\$ 5$ and $80 \%$ routine childhood coverage with mass vaccination of $50 \%$ of the $0-40$ age group to be preferred at vaccination costs from $\$ 5-25$. From $\$ 25-129$, mass vaccination of the $0-15$ age class was preferred, and between $\$ 129$ and $\$ 285$, mass vaccination of the $0-5$ age class was preferred. At vaccination costs beyond $\$ 285$, no vaccination at all was preferred to any of the five policies considered. $80 \%$ routine childhood coverage without mass vaccination never emerged as the preferred choice (Figure 3a).

When we sampled vaccination cost uniformly from $\$ 10-300$, we found that $80 \%$ routine coverage with one-time mass vaccination of the $0-5$ age class was preferred for willingnessto-pay values ranging from $\$ 13,500-\$ 30,200$, while $80 \%$ routine coverage with one-time mass vaccination of the $0-15$ age class was preferred from $\$ 30,200$ to beyond the costeffective threshold. These results demonstrate that cost-effectiveness is robust to vaccination cost (Figure 3b).

\section{Phase 2b dengue vaccine efficacy}

Using the dengue vaccine efficacy estimated in recent Phase $2 b$ trials, we found that herdimmunity cannot be achieved even with $100 \%$ routine childhood coverage. We also found vaccination may be cost-effective up to a cost of $\$ 237$ (95\% CI \$159-512), very costeffective up to a cost of $\$ 141$ (95\% CI \$63-416), and cost-saving up to a cost of $\$ 93$ (95\% CI \$15-368).

For the Phase $2 \mathrm{~b}$ vaccine efficacy, we found that $80 \%$ routine childhood coverage and onetime mass vaccination of $50 \%$ of the entire population was preferred at a vaccination cost up to $\$ 18,80 \%$ routine childhood coverage and one-time mass vaccination of $50 \%$ of the $0-40$ age class was preferred from $\$ 18-68$, and $80 \%$ routine childhood coverage and one-time mass vaccination of $50 \%$ of the $0-15$ age class was preferred at a vaccination cost from $\$ 68-121$. Beyond $\$ 121$, no vaccination at all was preferred to any of the five policies considered (Figure 4a). When we treated vaccination cost as an unknown parameter in the uniform range $\$ 10-300$, none of the five vaccination policies were preferred to no vaccination at all (Figure $4 b$ ).

\section{Conclusions and discussion}

Our results are pertinent to decisions of policymakers regarding the design of dengue vaccination policies. We demonstrated that, at an optimistic $70 \%$ vaccine efficacy, routine vaccination of $82 \%$ of young children is sufficient to maintain herd-immunity, as well as efficient and cost-effective across a range of vaccination costs. Achieving this level of vaccination coverage should be feasible logistically given that high levels of adherence to childhood vaccination schedules are consistently maintained in Brazil (26).

The herd-immunity threshold that we calculated provides a target range for maintaining protection of the population overall. Those policies with a level of routine coverage above the herd-immunity threshold are not cost-effective, because the excess routine coverage does little to further decrease dengue burden. Conversely, those policies with substantially lower levels of routine coverage do not adequately protect the population against dengue outbreaks, reducing the cost-effectiveness. However, policies that combine routine coverage with one-time mass vaccination of young age groups have the highest probability of having the greatest net benefits, by minimizing the lag time before population immunity is established. Therefore, our results highlight the importance of combining targeted mass 
vaccination as soon as the vaccine becomes available with routine herd-immunity coverage for optimal control and cost-effectiveness $(12,14,19)$.

While recent Phase $2 \mathrm{~b}$ trials demonstrated that the dengue vaccine is safe and confers some immunity against infection, the calculated vaccine efficacy of 30\% was less than expected (9). Our results suggest that even such a vaccine with $30 \%$ efficacy may be cost-effective and possibly cost-saving provided that the total vaccination cost can be kept sufficiently low, below $\$ 237$ for cost-effectiveness and below $\$ 93$ for cost-saving. However, the Phase $2 \mathrm{~b}$ trials showed no evidence of protection against DENV-2, which has been prevalent in Brazil in recent years $(9,29)$. Further vaccine trials are underway to validate and refine the efficacy estimates, as well as to resolve any sampling biases in the relatively small Phase $2 b$ trials (10). Should these initial efficacies remain consistent following further vaccine trials, our model may be extended to incorporate serotype-specific efficacy.

Concern about antibody-dependent enhancement (ADE), whereby prior infection with a single serotype of dengue enhances an individual's risk of severe infection with other serotypes, has motivated the development of tetravalent dengue vaccines that simultaneously protect against all four serotypes (5). The potential for a vaccine itself to generate ADE and result in more clinically severe disease has been cited by vaccine developers as a theoretical risk of vaccines currently under development, although the degree to which vaccines may generate ADE, if at all, has not yet been well established (7). Nonetheless, there is concern that ADE may enhance the risk of more severe dengue hemorrhagic fever as vaccine immunity wanes (6). Future expansions of our model could investigate the effect of waning vaccine immunity on population protection and, as more information becomes available about vaccine-induced ADE, quantify the tolerable range of ADE that maintains vaccination cost-effectiveness.

In this paper, we developed a host-vector model of dengue virus transmission in Brazil and used it to provide quantitative estimates of vaccine pricing, herd-immunity coverage levels, and conditions under which vaccination is likely to be cost-effective and cost-saving. Our results are in empirical agreement with observed levels of dengue prevalence $(2,27,28)$ and previous model predictions of necessary coverage for herd-immunity (17). While we focus on urban vaccination policies within Brazil, our model can be readily adapted to model other countries by calibrating the transmission and mosquito survival parameters to match regional endemic dengue prevalence.

Dengue continues to present a significant challenge in many regions of the world. We evaluated the cost-effectiveness of vaccination in light of recent dengue vaccine trial results. We showed that carefully targeted vaccination holds significant potential to confer excellent value and reduce the overall burden of dengue in Brazil.

\section{Supplementary Material}

Refer to Web version on PubMed Central for supplementary material.

\section{Acknowledgments}

We thank Ira Longini and Scott Halstead for helpful discussion. This research was funded by NIH grant U01GM087719. The funding source had no other involvement in the research. 


\section{References}

1. Suaya JA, Shepard DS, Siqueira JB, Martelli CT, Lum LCS, Tan LH, et al. Cost of dengue cases in eight countries in the Americas and Asia: a prospective study. The American Journal of Tropical Medicine and Hygiene. 2009 May; 80(5):846-55. [PubMed: 19407136]

2. Shepard DS, Coudeville L, Halasa YA, Zambrano B, Dayan GH. Economic impact of dengue illness in the Americas. American Journal of Tropical Medicine and Hygiene. 2011; 84(2):200-7. [PubMed: 21292885]

3. De Simone TS, Nogueira RMR, Araújo ESM, Guimarães FR, Santos FB, Schatzmayr HG, et al. Dengue virus surveillance: the co-circulation of DENV-1, DENV-2 and DENV-3 in the State of Rio de Janeiro, Brazil. Transactions of the Royal Society of Tropical Medicine and Hygiene. 2004 Sep; 98(9):553-62. [PubMed: 15251405]

4. De Melo FL, Romano CM, De Andrade Zanotto Pm. Introduction of dengue virus 4 (DENV-4) genotype I into Brazil from Asia? PLoS neglected tropical diseases. 2009 Jan.3(4):e390. [PubMed: 19399169]

5. Whitehead SS, Blaney JE, Durbin AP, Murphy BR. Prospects for a dengue virus vaccine. Nat Rev Micro. 2007; 5(7):518-28.

6. Schmitz J, Roehrig J, Barrett A, Hombach J. Next generation dengue vaccines: A review of candidates in preclinical development. Vaccine. 2011; 29(42):7276-84. [PubMed: 21781998]

7. Guy B, Barrere B, Malinowski C, Saville M, Teyssou R, Lang J. From research to phase III: preclinical, industrial and clinical development of the Sanofi Pasteur tetravalent dengue vaccine. Vaccine. 2011; 29(42):7229-41. [PubMed: 21745521]

8. Morrison D, Legg TJ, Billings CW, Forrat R, Yoksan S, Lang J. A novel tetravalent dengue vaccine is well tolerated and immunogenic against all 4 serotypes in flavivirus-naive adults. The Journal of Infectious Diseases. 2010; 201(3):370-7. [PubMed: 20059357]

9. Sabchareon A, Wallace D, Sirivichayakul C, Limkittikul K, Chanthavanich P, Suvannadabba S, et al. Protective efficacy of the recombinant, live-attenuated, CYD tetravalent dengue vaccine in Thai schoolchildren: a randomised, controlled phase $2 b$ trial. The Lancet. 2012 Sep 10; 380(9853):1559_ 67.

10. Halstead SB. Dengue vaccine development: a 75\% solution? Lancet. 2012 Nov 3; 380(9853): 1535-6. [PubMed: 22975339]

11. Zorlu, G.; Fleck, F. Bulletin of the World Health Organization. Vol. 89. World Health Organization; $2011 \mathrm{Jul}$ 1. Dengue vaccine roll-out: getting ahead of the game; p. 476-7.

12. Amarasinghe A, Mahoney RT. Estimating potential demand and supply of dengue vaccine in Brazil. Human vaccines. 2011 Jul; 7(7):776-80. [PubMed: 21734468]

13. Shepard DS, Suaya JA, Halstead SB, Nathan MB, Gubler DJ, Mahoney RT, et al. Costeffectiveness of a pediatric dengue vaccine. Vaccine. 2004; 22(9-10):1275-80. [PubMed: 15003657]

14. Shepard, DS.; Suaya, JA. Cost-Effectiveness of a Dengue Vaccine in Southeast Asia and Panama: Preliminary Estimates. In: Preedy, VR.; Watson, RR., editors. Handbook of Disease Burdens and Quality of Life Measures. New York, NY: Springer New York; 2010. p. 1281-96.

15. Lee BY, Connor DL, Kitchen SB, Bacon KM, Shah M, Brown ST, et al. Economic value of dengue vaccine in Thailand. The American Journal of Tropical Medicine and Hygiene. 2011; 84(5):764-72. [PubMed: 21540387]

16. Carrasco LR, Lee LK, Lee VJ, Ooi EE, Shepard DS, Thein TL, et al. Economic Impact of Dengue Illness and the Cost-Effectiveness of Future Vaccination Programs in Singapore. PLoS Neglected Tropical Diseases. 2011; 5(12):e1426. [PubMed: 22206028]

17. Johansson MA, Hombach J, Cummings DAT. Models of the impact of dengue vaccines: A review of current research and potential approaches. Vaccine. 2011; 29(35):5860-8. [PubMed: 21699949]

18. Ibuka Y, Paltiel AD, Galvani AP. Impact of program scale and indirect effects on the costeffectiveness of vaccination programs. Medical decision making? : an international journal of the Society for Medical Decision Making. 2012 May 1; 32(3):442-6. [PubMed: 22472916] 
19. Mahoney RT, Francis DP, Frazatti-Gallina NM, Precioso AR, Raw I, Watler P, et al. Cost of production of live attenuated dengue vaccines: a case study of the Instituto Butantan, Sao Paulo, Brazil. Vaccine. 2012 Jul 6; 30(32):4892-6. [PubMed: 22406455]

20. Gold, MR.; Siegel, JE.; Russell, LB.; Weinstein, MC. editors Cost-effectiveness in health and medicine. New York: Oxford University Press; 1996.

21. CIA. CIA - The World Factbook - Brazil [Internet]. [cited 2012 Feb 24]. Available from: https:// www.cia.gov/library/publications/the-world-factbook/geos/br.html

22. Stinnett AA, Mullahy J. Net Health Benefits A New Framework for the Analysis of Uncertainty in Cost-Effectiveness Analysis. Medical Decision Making. 18(2):S68-S80.

23. World Bank. GDP per capita [Internet]. Available from: http://data.worldbank.org/indicator/ NY.GDP.PCAP.CD

24. IMF. World Economic Outlook Database List [Internet]. Available from: http://www.imf.org/ external/pubs/ft/weo/2012/02/weodata/index.aspx

25. WHO. Report of the Commission on Macroeconomics and Health. Geneva: Macroeconomics and health: investing in health for economic development.

26. Barata RB, Sampaio de Almeida Ribeiro MC, De Moraes JC, Flannery B. Socioeconomic inequalities and vaccination coverage: results of an immunisation coverage survey in 27 Brazilian capitals, 2007-2008. Journal of epidemiology and community health. 2012 Oct; 66(10):934-41. [PubMed: 22268129]

27. Cordeiro MT, Silva AM, Brito CAA, Nascimento EJM, Magalhaes MCF, Guimaraes GF, et al. Characterization of a Dengue Patient Cohort in Recife, Brazil. Am J Trop Med Hyg. 2007 Dec 1; 77(6):1128-34. [PubMed: 18165535]

28. Balmaseda A, Hammond SN, Tellez Y, Imhoff L, Rodriguez Y, Saborío SI, et al. High seroprevalence of antibodies against dengue virus in a prospective study of schoolchildren in Managua, Nicaragua. Tropical medicine \& international health. 2006 Jun; 11(6):935-42. [PubMed: 16772016]

29. Oliveira MF, Galvao Araujo JM, Ferreira OC, Ferreira DE, Lima DB, Santos FB, et al. Two lineages of dengue virus type 2, Brazil. Emerging infectious diseases. 2010 Mar; 16(3):576-8. [PubMed: 20202456] 


\section{Highlights}

- We model dengue vaccination and cost-effectiveness under two efficacy scenarios.

- We calculate the level of coverage necessary to sustain population herd immunity.

- We examine vaccination cost-effectiveness under 400 unique roll-out policies.

- We quantify the price below which vaccination is cost-effective and is costsaving.

- We evaluate the sensitivity of our results to parameter and price uncertainty. 


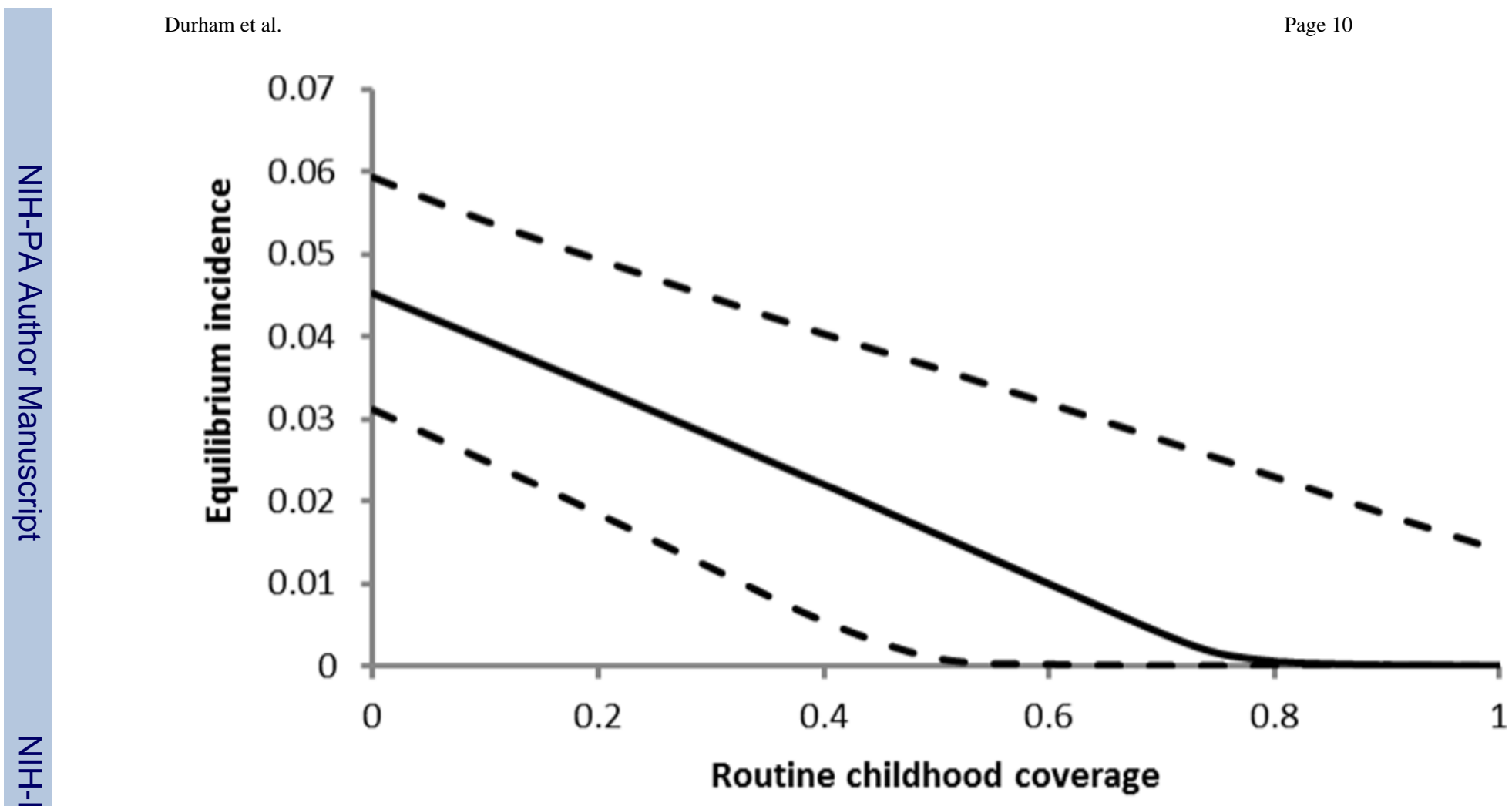

Figure 1.

Annual endemic dengue incidence (symptomatic and asymptomatic) as the routine childhood coverage at 70\% vaccine efficacy increases, with 95\% credible intervals (dashed). 
a)

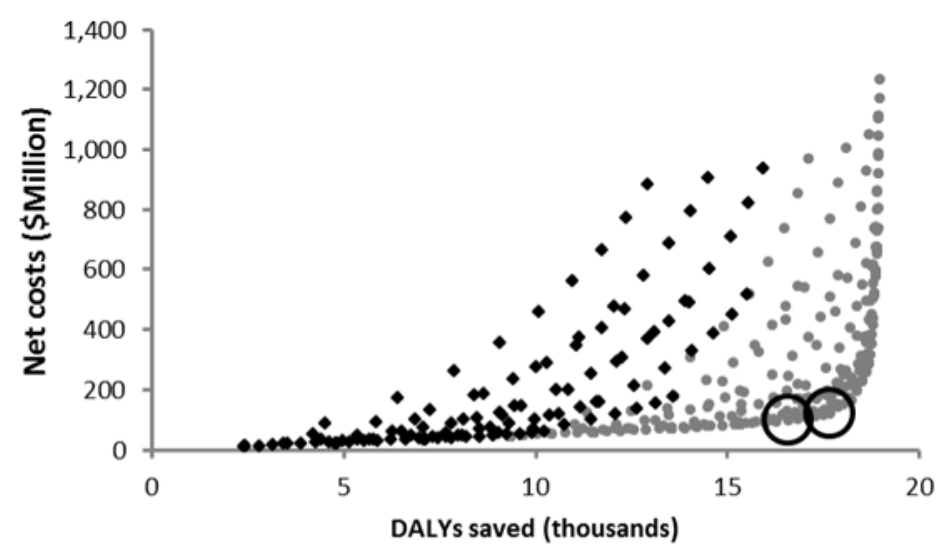

b)

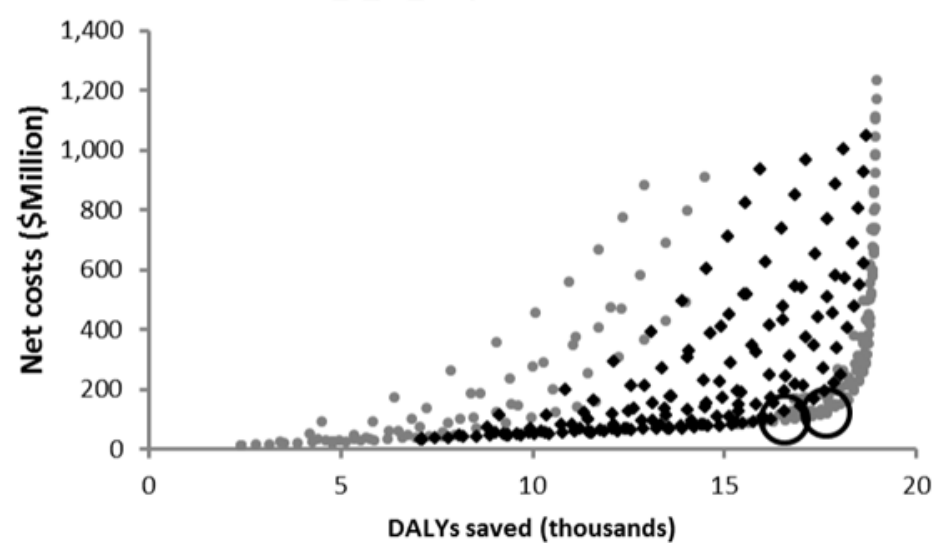

Figure 2.

Net cost and DALYs saved across 400 vaccination policies at a vaccination cost of $\$ 250$. Each point represents a distinct policy combining between 0 and $90 \%$ routine childhood coverage with one-time mass vaccination of between 0 and $90 \%$ of age groups $0-5$ year olds, 0-15 year olds, 0-40 year olds, or the full population. Bold diamonds indicate those policies that include a) 0-30\% routine coverage, b) 30-60\% routine coverage, c) $60-80 \%$ routine coverage, and d) $80-90 \%$ routine coverage. The largest policies that are very costeffective and cost-effective are circled. Net cost and DALYs saved were discounted and summed over a 73 year period (the average life expectancy in Brazil). 
c)

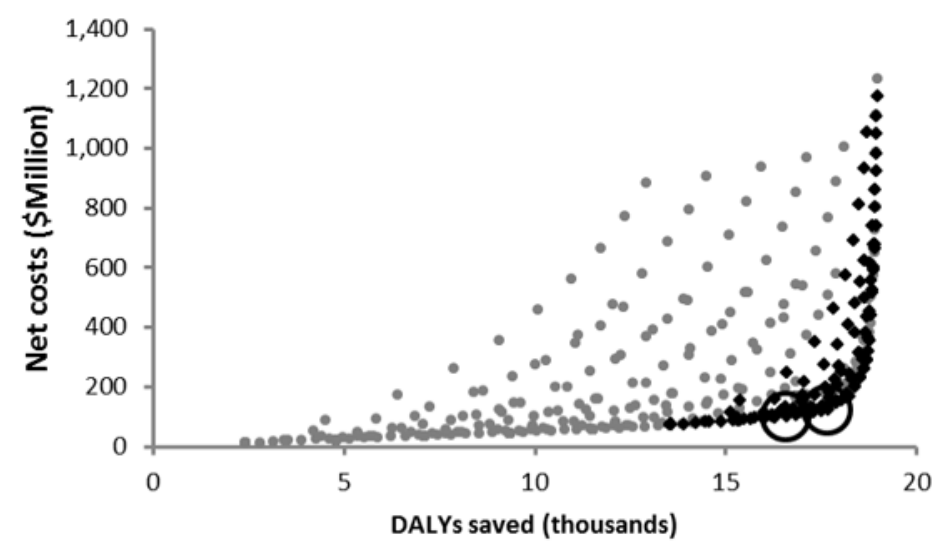

d)

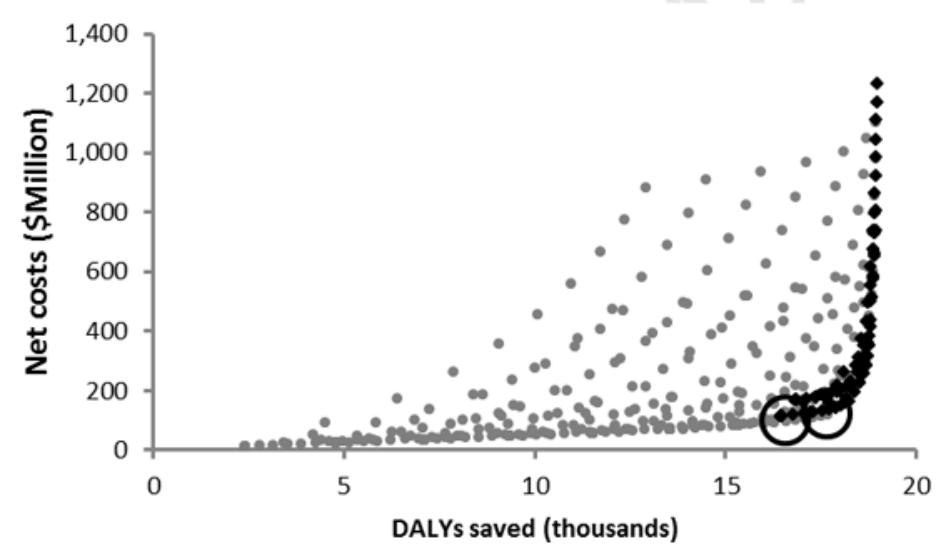

Figure 3.

Preferred policies, having the highest probability of having the greatest net benefits, at $70 \%$ vaccine efficacy. a) Preferred policies, at a willingness-to-pay of US \$36,000, as vaccination cost increases. b) Preferred policies with vaccination costs sampled uniformly from the range (\$10,\$300), as willingness-to-pay increases. Vertical dashed lines indicate the very cost-effective threshold (US \$12,000) and the cost-effective threshold (US \$36,000).

Evaluated policies include progressively more extensive mass vaccination: 1) No vaccination, 2) $80 \%$ routine childhood coverage with no mass vaccination, 3) $80 \%$ routine childhood coverage with mass vaccination of $50 \%$ of $0-5 \mathrm{yr}$ olds, 4) $80 \%$ routine childhood coverage with mass vaccination of $50 \%$ of $0-15 \mathrm{yr}$ olds, 5) $80 \%$ routine childhood coverage with mass vaccination of $50 \%$ of $0-40$ year olds, and 6) $80 \%$ routine childhood coverage with mass vaccination of $50 \%$ of the full population. 
a)

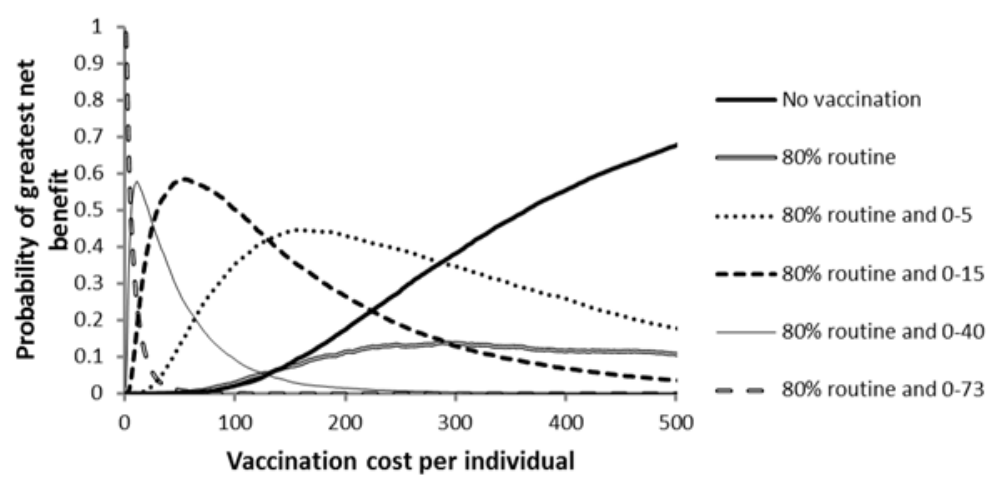

b)

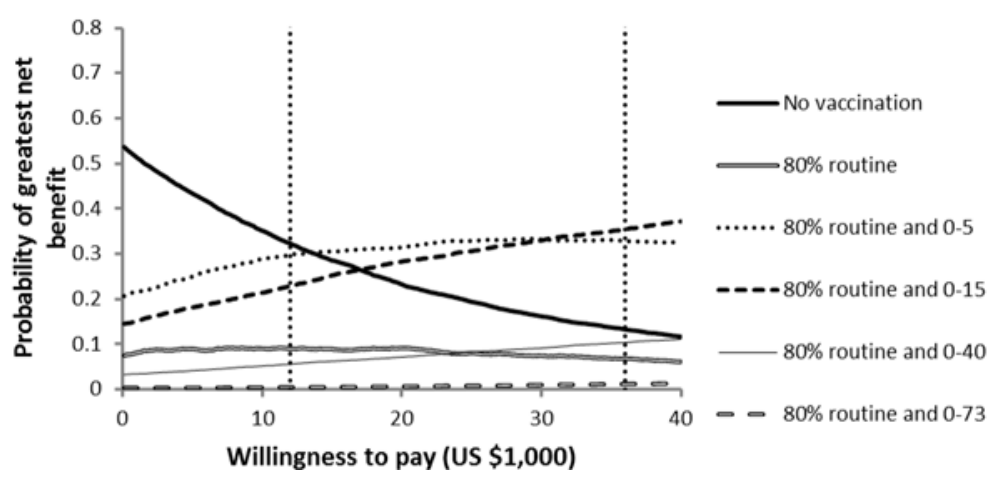

Figure 4.

Preferred policies, having the highest probability of having the greatest net benefits, at $30 \%$ vaccine efficacy. a) Preferred policies at a willingness-to-pay of US \$36,000, as vaccination cost increases. b) Preferred policies with vaccination costs sampled uniformly from the range $(\$ 10, \$ 300)$, as willingness-to-pay increases. Vertical dashed lines indicate the very cost-effective threshold (US \$12,000) and the cost-effective threshold (US \$36,000). 